\title{
Protective Effect of Resveratrol Against Cadmium- Induced Toxicity On Ovine Oocyte In Vitro Maturation And Fertilization
}

\section{Anna Rita Piras}

University of Sassari

\section{Federica Ariu}

University of Sassari

Alessio Maltana

University of Sassari

Giovanni Giuseppe Leoni

University of Sassari

Nicola Antonio Martino

University of Bari Aldo Moro

Antonella Mastrorocco

University of Bari Aldo Moro

Maria Elena Dell'Aquila

University of Bari Aldo Moro

Luisa Bogliolo ( $\sim$ luis@uniss.it )

University of Sassari https://orcid.org/0000-0003-3205-7362

\section{Research Article}

Keywords: Oocyte, cadmium, in vitro maturation, resveratrol, ovine

Posted Date: February 8th, 2022

DOI: https://doi.org/10.21203/rs.3.rs-1299295/v1

License: (c) (1) This work is licensed under a Creative Commons Attribution 4.0 International License.

Read Full License 
Protective effect of resveratrol against cadmium-induced toxicity on ovine oocyte in vitro maturation and fertilization

Anna Rita Piras ${ }^{1}$, Federica Ariu ${ }^{1}$, Alessio Maltana ${ }^{1}$, Giovanni Giuseppe Leoni ${ }^{1}$, Nicola Antonio Martino $^{2}$, Antonella Mastrorocco ${ }^{2}$, Maria Elena Dell'Aquila $^{2}$, Luisa Bogliolo ${ }^{1}$

${ }^{1}$ Department of Veterinary Medicine, University of Sassari, 017100, Sassari, Sardinia, Italy.

${ }^{2}$ Department of Biosciences, Biotechnologies \& Biopharmaceutics, University of Bari Aldo Moro, 70125 Bari, Italy.

Corresponding Author: Luisa Bogliolo, luis@uniss.it

AR Piras : anrpiras@uniss.it

F Ariu : federica@uniss.it

A Maltana: alessio.maltana@gmail.com

GG Leoni: gioleoni@uniss.it

NA Martino: nicola.martino@uniba.it

A Mastrorocco: antonella.mastrorocco@uniba.it

ME Dell'Aquila: mariaelena.dellaquila@uniba.it

LBogliolo: luis@uniss.it

Key words: Oocyte, cadmium, in vitro maturation, resveratrol, ovine 


\section{Abstract}

Background: Heavy metal cadmium $(\mathrm{Cd})$ is a widespread environmental contaminant with a potential toxicity that might negatively affect female reproduction and fertility. It has been reported that Cd exposure impaired the quality of oocytes and led to a defective maturation and fertilization, through oxidative stress induction. Resveratrol (Res) is a natural polyphenol with strong antioxidant properties that exhibited protective role in preventing oocyte redox homeostasis disruption and quality decline. Here, we explored whether the addition of Res to in vitro maturation (IVM) medium might act as a protection against Cd-induced toxicity on ovine oocyte maturation and fertilization. Firstly, we evaluated the effect of supplementing IVM medium with two different Res concentrations ( 1 and $2 \mu \mathrm{mol} / \mathrm{L})$ on nuclear maturation and fertilization of oocytes matured under CdCl2 $(2 \mu \mathrm{mol} / \mathrm{L})$ exposure. Therefore, the concentration of $1 \mu \mathrm{mol} / \mathrm{L}$ Res was selected to analyse intracellular ROS levels, mitochondrial (mt) distribution and activity, chromatin configuration, cytoskeleton morphology, cortical granules (CGs) distribution and mRNA expression of genes associated with cellular response to oxidative stress (i.e. SIRT1, SOD 1, GPX1, GSR, CAT) in Cd-exposed in vitro matured oocytes.

Results: We found that $1 \mu \mathrm{mol} / \mathrm{L}$ Res was effective to recover the reduced oocyte meiotic competence induced by Cd exposure, while treatment with Res, at both tested concentrations, sustained oocyte ability to be normally fertilized and decreased polyspermic fertilization. Moreover, we demonstrated that $1 \mu \mathrm{mol} / \mathrm{L}$ Res mitigated Cd-induced alterations of oocyte cytoplasmic maturation by reducing reactive oxygen species (ROS) accumulation, preventing mt dysfunction, maintaining the correct meiotic spindle and cortical F-actin assembly and the normal cortical granule distribution as well as up-regulating SIRT1, SOD1 and GPX1 genes.

Conclusions: Taken together, our findings highlighted the beneficial influence exerted by Res in preventing $\mathrm{Cd}$-induced disturbance of nuclear and cytoplasmic maturation and subsequent fertilization in ovine oocytes. Res treatment may help to establish defence strategies counteracting Cd-induced toxicity on the female gamete. 


\section{Background}

Anthropogenic activities and climate change have led to a dramatic increase of chemical pollutants levels in terrestrial and aquatic ecosystems. Over recent years, a large body of studies have underlined associations between the exposure to several environmental chemicals and female reproductive disorders in humans and animals $[1,2]$.

Among inorganic pollutants, heavy metals represent a potential threat to reproductive health, due to the high global annual emission rate. $\mathrm{Cd}$ is considered one of the most toxic nonessential heavy metal that is widely distributed in air, water and soil. Anthropogenic $\mathrm{Cd}$ emissions arise from industrial processes including the combustion of fossil fuels, waste incineration, smelting and mining, rubber processing, manufacturing of nickel-Cd batteries, plastic stabilizer and pigment [3]. This metal can be found in phosphate fertilizers [4] and it is a major component of cigarette smoke [5]. Given its low excretion from the body and its long biologic half-life (15-30 years), Cd accumulates and remains in organ and tissues over time [6].

Bioaccumulation of $\mathrm{Cd}$ has been found in the ovaries and follicular fluid of both humans and animals [7-10]. This metal was detected as the trace element with the highest age-dependent ovarian bioaccumulation in sheep [8].

Cadmium toxicity on female reproductive system has been largely explored in vivo in laboratory animal models [11-15]. Some of the adverse effect of cadmium exposure include interference with the hypothalamic-pituitary-ovarian axis, reduced steroidogenesis, inhibition of follicle and oocyte development, impairment of ovulation and oocyte pick-up by the tubal epithelium together with retardation of embryo development and implantation, restricted fetal growth and pregnancy complications [16].

By using different in vitro mammalian models, previous studies pointed out the negative impact of Cd exposition on oocyte meiotic competence during in vitro maturation (IVM) and on their ability to successfully undergo in vitro fertilization (IVF) and support preimplantation embryo development. In detail, the meiotic progression of ovine, bovine, buffalo and porcine oocytes [17-22] was 
significantly reduced following Cd exposure during IVM. Besides, other evidences proved that in vivo and in vitro $\mathrm{Cd}$ exposure caused defects of cytoplasmic maturation in mouse and porcine oocytes, including disruption of spindle formation and chromosome alignment, impaired polymerization of actin on the plasma membrane, aberrant distribution of mitochondria (mt) and cortical granules (CGs) as well as changes of epigenetic modifications [10,22]. The cytotoxic effect of Cd on IVM resulted in a decline of fertilization rates and an increase of polyspermy in ovine oocytes $[8,21]$ while a reduced sperm binding ability was recorded in porcine oocytes [22]. Most of the abovementioned studies were performed using $\mathrm{Cd}$ concentration in the micromolar range. Previously, we evaluated the effect of exposure to $\mathrm{Cd}$ during in vitro maturation at environmental nanomolar concentrations on the fertilization rates of oocytes from juvenile and adult sheep [8]. We found that, even at such a low Cd concentration, the IVF rate was significantly reduced and there was also a slight increase in the number of abnormally fertilized oocytes. More interestingly, our results indicated that Cd caused mitochondria dysfunction, increased reactive oxygen species (ROS) levels and lipid peroxidation which negatively affected the oocyte ability to be fertilized.

In agreement with our findings [22], demonstrated that $\mathrm{Cd}$ exposure caused an excessive increase of ROS levels that led to DNA damage and apoptosis and to a defective IVM and IVF of porcine oocytes. Furthermore, female mice exposure to chronical and acute $\mathrm{Cd}$ impaired oocyte meiotic progression and decreased fertility by enhancing ROS level and apoptosis [12, 13].

Based on the role of oxidative stress in Cd reproductive toxicity, developing defence strategies using antioxidants is a growing field of study [23]. Glutathione supplementation during IVM of porcine oocytes was able to restore Cd-exposure causing meiotic defects and affecting oocyte quality via eliminating the excessive ROS [22]. Furthermore, the beneficial role exerted by melatonin in preventing Cd-induced oxidative stress in ovaries in rats has been recently highlighted [24].

Resveratrol (Res; 3,4,5-trihydroxy-trans-stilbene), a phytoalexin produced by plants, is one of the most studied polyphenol with well-established antioxidant properties [25]. Several studies provided evidence that Res could act as a powerful antioxidant being able to prevent the disruption of oocyte 
redox homeostasis and the decline of the oocyte quality. This antioxidant improved oocyte IVM and IVF and subsequent embryonic development in various species [26-30]. In addition, administration of Res effectively protected against postovulatory mouse oocyte aging both in vivo and in vitro primarily by preventing ROS production and by improving mitochondrial function [31-33]. It has also been shown that Res enhanced the resistance of oocytes in response to sub-optimal conditions including the exposition to toxic chemicals [34, 35], heat and hypothermic stresses [30, 36] and cryopreservation $[37,38]$.

Our study aimed at exploring the effect of supplementing maturation medium with Res on IVM and IVF of ovine oocytes in vitro cultured under Cd-exposure. Given the positive influence of Res observed, the effect of Res on the quality of Cd-exposed in vitro matured oocyte was also investigated by assessing intracellular ROS levels, mitochondrial distribution and activity, chromatin configuration, cytoskeleton morphology, cortical granules (CGs) distribution and mRNA expression of genes associated with cellular response to oxidative stress (i.e. SIRT1, SOD 1, GPX1, GSR, CAT).

\section{Methods}

\section{Chemicals}

Unless otherwise specified, all chemicals were purchased from Sigma-Aldrich (Milan, Italy).

\section{Oocyte collection}

Ovaries were collected from slaughtered juvenile Sarda ewes and transported to the laboratory within $3 \mathrm{~h}$ in phosphate-buffered saline (PBS) with antibiotics at $37^{\circ} \mathrm{C}$.

Cumulus oocytes complexes (COCs) were retrieved by slicing procedure in dissection medium (DM) consisting of $20 \mathrm{mmol} / \mathrm{L}$ Hepes-buffered TCM 199 supplemented with polyvinyl alcohol (0 1\%, w/v) and antibiotics. COCs with two or more complete layers of compact cumulus cells and with homogeneous cytoplasm were selected for IVM. 


\section{In vitro maturation and in vitro fertilization (IVF)}

Groups of COCs were matured in vitro in TCM 199 supplemented with heat-treated oestrous sheep serum $(10 \%$, OSS $)$, pyruvate $(0.36 \mu \mathrm{mol} / \mathrm{L})$, cysteamine $(100 \mu \mathrm{mol} / \mathrm{L})$, Follicle-Stimulating Hormone (FSH; $1 \mathrm{IU} / \mathrm{mL})$, Luteinizing Hormone (LH; $1 \mathrm{IU} / \mathrm{mL}$ ) under mineral oil, in four-well dishes (Nunc Cell Culture, Thermo Fisher Scientific, Waltham, Massachusetts, USA) in a humidified atmosphere of $5 \% \mathrm{CO}_{2}$, at $38.5^{\circ} \mathrm{C}$ for $24 \mathrm{~h} \mathrm{[39].}$

After IVM, COCs were completely denuded of granulosa cells via gentle pipetting with a fine bore glass pipette. Oocytes at the metaphase II (MII) stage were selected under a stereomicroscope (Olympus SZ-PT, Italy) for the presence of the first polar body and randomly assigned to the analyses. For IVF groups of MII oocytes from the different experimental groups were co-incubated with frozenthawed ram spermatozoa $\left(1 \times 10^{6}\right.$ spermatozoa/mL), selected by the swim-up technique in Synthetic Oviductal Fluid (SOF, [40]) supplemented with OSS (2\%), heparin $(1 \mu \mathrm{g} / \mathrm{mL})$, hypotaurine $(1 \mu \mathrm{g} / \mathrm{mL})$ for $16 \mathrm{~h}$ in a humidified atmosphere of $5 \% \mathrm{CO}_{2}$ at $38.5^{\circ} \mathrm{C}$ [39].

\section{Fertilization assessment}

At the end of IVF, the presumptive zygotes were fixed in ethanol absolute with Hoechst 33258 (10 $\mu \mathrm{g} / \mathrm{mL}$ ) at $4{ }^{\circ} \mathrm{C}[41]$, mounted on microscope slides covered with cover slips and observed under an inverted fluorescence microscope (Olympus IX70, Italy). Fertilization assessment was performed as follows [8]: -normal fertilization: male and female pronuclear formation (2PN) and extrusion of two polar bodies (PBs);

-polyspermic fertilization: more than two pronuclei (>2PN) and two PBs;- asynchronous fertilization: one PN and a sperm head (SH) that failed chromatin decondensation and two PBs or one PN and MII plate and two PBs; -unfertilized oocytes : MII plate and first PB. 


\section{Determination of ROS level}

MII oocytes were incubated for $30 \mathrm{~min}$ in PBS with bovine serum albumin (3\%, BSA) containing $10 \mu \mathrm{mol} / \mathrm{L} 2^{\prime} 7^{\prime}$ - dichlorodihydrofluorescein diacetate $\left(\mathrm{H}_{2} \mathrm{DCF}-\mathrm{DA}\right)$ at $38.5^{\circ} \mathrm{C}, 5 \% \mathrm{CO}_{2}$ in air, in order to detect the dichlorofluorescein (DCF) and localize intracellular sources of ROS [28]. After three times wash in PBS/BSA, oocytes were fixed overnight at $4^{\circ} \mathrm{C}$ with $2 \%$ paraformaldehyde (PFA) solution in PBS. The oocytes were mounted on glass slides with $10 \mu \mathrm{g} / \mathrm{mL}$ Hoechst 33258 in PBS and glycerol solution $(3: 1, \mathrm{v} / \mathrm{v})$ and fluorescence was detected by a laser-scanning confocal microscope (LSCM, Leica TCS SP5) with LAS AF Lite 170 image analysis software package (Leica Microsystems GmbH, Wetzlar, Germany). An argon ions laser ray at $488 \mathrm{~nm}, 495 \mathrm{~nm}$ (excitation) and $520 \mathrm{~nm}$ (emission) filters were used to detect the DCF fluorescence intensity.

\section{Evaluation of mitochondrial activity and distribution}

MII oocytes underwent a staining with MitoTracker Orange CMTM Ros (Molecular Probes, Inc., Eugene, OR, USA) in order to detect active mt distribution and mt act [28]. Briefly, the oocytes were incubated for $30 \mathrm{~min}$ in PBS /BSA containing $280 \mathrm{nmol} / \mathrm{L}$ MitoTracker Orange CMTM Ros, at $38.5^{\circ} \mathrm{C}, 5 \% \mathrm{CO}_{2}$ in air. The oocytes were mounted on glass slides with $10 \mu \mathrm{g} / \mathrm{mL}$ Hoechst 33258 in PBS and glycerol solution and imaged under TCS SP5 LSCM. A helium/neon laser ray at 543 nm, $551 \mathrm{~nm}$ (excitation) and $576 \mathrm{~nm}$ (emission) filters were used to point out the MitoTrackerOrange CMTM Ros. LSCM settings were kept constant for all experiments.

In each individual oocyte MitoTracker fluorescence intensities were measured on the section corresponding to the equatorial plane of the oocyte as described previously [28].

A homogeneous mt distribution, with small granulations spread throughout the cytoplasm was considered as the normal mt distribution pattern [42]. The percentage of oocytes with normal and abnormal mt distribution was calculated in each group. 
Immunofluorescence detection of cytoskeletal structures (meiotic spindle, cortical F-actin)

MII oocytes were fixed in microtubule-stabilising buffer $(100 \mathrm{mmol} / \mathrm{L} \mathrm{PIPES,} 5 \mathrm{mmol} / \mathrm{L} \mathrm{MgCl} 2,2.5$ mol/L EGTA, $2 \%$ formaldehyde, $0.1 \%$ Triton X-100, $1 \mathrm{~mol} / \mathrm{L}$ taxol, $10 \mathrm{U} / \mathrm{ml}$ aprotinin and $50 \%$ deuterium oxide) for $1 \mathrm{~h}$ at $37{ }^{\circ} \mathrm{C}$ and stored in blocking solution $(0.2 \%$ sodium azide, $2 \%$ normal goat serum, $1 \% \mathrm{BSA}, 0.1 \mathrm{~mol} / \mathrm{L}$ glycine and $0.1 \%$ Triton $\mathrm{X}-100$ in PBS) at $4{ }^{\circ} \mathrm{C}$ until processing [43]. Samples were incubated overnight at $4^{\circ} \mathrm{C}$ with a mixture of mouse monoclonal anti $\alpha$-tubulin (dilution 1:1000), and mouse monoclonal anti $\beta$-tubulin (dilution 1:100) antibodies followed by incubation with donkey anti-mouse Alexa Fluor 488 (dilution 1:100; Life Technologies, Invitrogen, Carlsbad, California, USA) combined with rhodamine-phalloidin (dilution 1:150; Invitrogen, Carlsbad, CA) for $1 \mathrm{~h}$ at room temperature. DNA was stained with Hoechst $33258(10 \mu \mathrm{g} / \mathrm{mL})$. Images of chromatin, meiotic spindles, and cortical F-actin were acquired by LSCM using an oil immersion 40x objective and recorded on a host computer. Oocytes were scanned through the Z-axis and the images of the F-actin were recorded on the section corresponding to the equatorial plane of the cell.

A symmetrical barrel-shaped spindle structure with aligned chromosomes at the equatorial plate was defined as the normal spindle and chromatin configuration $[43,44]$.

An evenly stained layer of F-actin band immediately beneath the plasma membrane was considered as a normal actin distribution $[43,45]$.

The proportion of oocytes with normal and abnormal spindle, chromosome and cortical F-actin configuration was counted in each group.

\section{Assessment of CGs distribution}

Analysis of CGs was performed according to the methodology of Hosseini et al. [46]. MII oocytes were incubated with pronase $(0.5 \%)$ at $37^{\circ} \mathrm{C}$ to remove the zona pellucida and fixed with PFA $(2 \%)$ for $30 \mathrm{~min}$ at room temperature. After fixation, oocytes were permeabilized in PBS with Triton X$100(0.1 \%)$ for $5 \mathrm{~min}$, and then incubated in PBS with lectin peanut agglutinin (100 $\mu \mathrm{g} / \mathrm{mL}$, PNA) 
Alexa Fluor 488-conjugated (Molecular probes, Invitrogen) for $30 \mathrm{~min}$ at $37^{\circ} \mathrm{C}$. After three washes in PBS with BSA (0.3\%), glycine (100-mmol/L) and Triton X-100 (0.01\%), DNA was stained with Hoechst $33258(10 \mu \mathrm{g} / \mathrm{mL})$ and oocytes were mounted on glass slides overlaid with a coverslip. The distribution of CGs was examined at the equatorial plane of the oocytes by LSCM (490-nm excitation wavelength). CGs localized in the cortical cytoplasm of the oocytes were considered as a normal pattern of distribution $[47,48]$. The proportion of oocytes with normal and abnormal CGs distribution was counted.

\section{RNA extraction and quantitative real-time RT-PCR}

Three groups of fifteen MII oocytes each were analysed for each experimental conditions. Oocytes were snap frozen in liquid nitrogen and stored at $-80^{\circ} \mathrm{C}$ until further analysis. Total RNA was isolated from oocytes with RNeasy Micro Kit (Qiagen, Hilden,Germany) following manufacturer's instructions. Extracted RNA was treated with DNase I to exclude any potential genomic DNA contamination. The RNA isolated from oocytes was entirely and immediately used for reverse transcription-polymerasechain reaction (RT-PCR). The High Capacity Complementary DNA (cDNA) Reverse Transcription kit (Life Technologies, Monza, Italy) was used to convert RNA to cDNA. Briefly, the total RNA of each sample was added to $2 \mu \mathrm{L} 10 \mathrm{x}$ RT buffer, $0.8 \mu \mathrm{L} 25 \mathrm{x}$ dNTP mix, $2 \mu \mathrm{L}$ RT random primers, $1 \mu \mathrm{L}$ M-MLV RT, $1 \mu \mathrm{L}$ RNase inhibitor, and nuclease-free $\mathrm{H} 2 \mathrm{O}$ for a total volume of $20 \mu \mathrm{L}$ and incubated at $10^{\circ} \mathrm{C}$ for 10 minutes, then at $37{ }^{\circ} \mathrm{C}$ for 120 minutes, and finally at $85^{\circ} \mathrm{C}$ for 5 minutes. The relative quantification of the transcripts was carried out by Real Time RT-PCR with the StepOne instrument (Applied Biosystems, Foster City, CA, USA) using specific primers for each gene (Table 1). RT-PCR was performed in $20 \mu \mathrm{L}$ reaction volume containing: $10 \mu \mathrm{L}$ PowerUp SYBR Green PCR Master Mix (Applied Biosystems, 2x), $1 \mu \mathrm{L}$ of each primer $(200 \mathrm{nmol} / \mathrm{L}), 1 \mu \mathrm{L}$ of cDNA and nuclease-free water up to $20 \mu \mathrm{L}$. The temperature protocol consisted in $2^{\prime}$ at $95^{\circ} \mathrm{C}$ followed by 40 cycles at $94{ }^{\circ} \mathrm{C}(45 \mathrm{~s}), 60^{\circ} \mathrm{C}(45 \mathrm{~s})$ and $72{ }^{\circ} \mathrm{C}(45 \mathrm{~s})$. A melting curve was finally performed to detect PCR specificity. Relative quantification was performed by 
using the 2- $\Delta \Delta \mathrm{Ct}$ method according to Livak and Schmittgen [49] using the $\beta$-actin as housekeeping gene.

Table 1 Information of primers used in RT-qPCR

\begin{tabular}{|c|c|c|c|c|}
\hline Symbol & Gene name & Accession ID & Primer Seq $\left(5^{\prime} \rightarrow 3^{\prime}\right)$ & $\mathbf{T m},{ }^{\circ} \mathrm{C}$ \\
\hline SIRT1 & Sirtuin 1 & XM_012125645.2 & $\begin{array}{l}\text { AGTTGGTGATGGACAGGGAG } \\
\text { CGCTAGTTCAGTTCAGTCGC }\end{array}$ & $59^{\circ}$ \\
\hline SOD1 & $\begin{array}{l}\text { Superoxide } \\
\text { dismutase } 1\end{array}$ & NM_001145185 & $\begin{array}{l}\text { GGCAATGTGAAGGCTGACAA } \\
\text { TGCCCAAGTCATCTGGTCTT }\end{array}$ & $59^{\circ}$ \\
\hline$G P X 1$ & $\begin{array}{l}\text { Glutathione } \\
\text { peroxidase } 1\end{array}$ & GAAI01007125 & $\begin{array}{l}\text { ACCCAGATGAATGACCTGCA } \\
\text { TCGGACGTACTTCAGGCAAT }\end{array}$ & $59^{\circ}$ \\
\hline$G S R$ & $\begin{array}{l}\text { Glutathione- } \\
\text { disulfide reductase }\end{array}$ & XM_015104590 & $\begin{array}{l}\text { CTGCCCTGGGTTCTAAGACA } \\
\text { ACCTGGGAGTACTTCAGCAC }\end{array}$ & $59^{\circ}$ \\
\hline$C A T$ & Catalase & GQ421282 & $\begin{array}{l}\text { ACGCCTGTGTGAGAACATTG } \\
\text { AGCCATACTCAGGATGGACA }\end{array}$ & $59^{\circ}$ \\
\hline$A C T B$ & Actin B & NM_001009784 & $\begin{array}{l}\text { CCCTGGAGAAGAGCTACGAG } \\
\text { TAGTTTCGTGAATGCCGCAG }\end{array}$ & $59^{\circ}$ \\
\hline
\end{tabular}

\section{Experimental design}

Experiment 1: firstly, our aim was to evaluate the effects of supplementing IVM medium with two different Res concentrations on the nuclear maturation and fertilization rates of oocytes matured under cadmium (Cadmium chloride, $\mathrm{CdCl}_{2}$ ) exposure.

To this end, COCs were randomly divided in four groups and cultured in IVM medium supplemented with $0 \mu \mathrm{mol} / \mathrm{L} \mathrm{CdCl}_{2}$ (control, Ctr-group), $2 \mu \mathrm{mol} / \mathrm{L} \mathrm{CdCl}_{2}$ (Cd-group), $2 \mu \mathrm{mol} / \mathrm{L} \mathrm{CdCl}_{2}+1 \mu \mathrm{mol} / \mathrm{L}$ Res (Cd Res 1-group) and $2 \mu \mathrm{mol} / \mathrm{L} \mathrm{CdCl}{ }_{2}+2 \mu \mathrm{mol} / \mathrm{L}$ Res (Cd Res 2- group). 
After IVM, oocytes from the different experimental groups underwent IVF, followed by the evaluation of the percentage of normally and abnormally fertilized oocytes.

The concentration of $2 \mu \mathrm{mol} / \mathrm{L} \mathrm{Cd}$ was selected because it represents an intermediate concentration compared with those previously used in in vitro Cd-toxicity studies in various animal models [17-19, 21]. In addition, in a previous report [17], we observed that the exposure of ovine oocytes to $2 \mu \mathrm{mol} / \mathrm{L}$ $\mathrm{Cd}$ reduced the maturation and fertilization rate and increased polyspermy whereas higher concentrations exhibited lethal toxicity.

$\mathrm{CdCl}_{2}$ was dissolved in PBS (10 mmol/L $\mathrm{CdCl}_{2}$ stock solution) and diluted in TCM199 medium to a final concentration of $2 \mu \mathrm{mol} / \mathrm{L}$ immediately before culture.

Experiment 2: Based on the results of experiment 1, we explored the effect of Res supplementation ( $1 \mu \mathrm{mol} / \mathrm{L})$ during IVM on the quality of Cd-exposed in vitro matured oocyte by evaluating intracellular ROS levels, the $\mathrm{mt}$ distribution and activity, the chromatin configuration and morphology of meiotic spindle and cortical F-actin, the distribution of CGs, as well as, the mRNA expression of SIRT1, SOD 1, GPX1, GSR and CAT genes.

\section{Statistical analysis}

All statistical analyses were performed using STATAIIC 11.28 (StataCorp LP, Lakeway Drive, college station, TX, USA). Data on IVM, IVF, mt, CGs, chromatin and cytoskeleton were analyzed by chi-square test, through the Fisher's exact post-hoc test conducted to identify differences among the experimental classes.

The Shapiro-Wilk test was used to verify the normal distribution of data on intracellular ROS level, mt activity and gene expression. Parametric Analysis of Variance (ANOVA) with Bonferroni correction was made to compare normally distributed data. A post-hoc Tukey test was used to explore difference among experimental groups when data were statistically different. When not normally distributed, a non-parametric Kruskal-Wallis test was used.

Differences with $P<0.05$ were considered statistically significant. All experiments were replicated at least three times. 


\section{Results}

\section{Experiment 1}

\section{The effect of Res on nuclear maturation and fertilization in Cd-exposed oocytes}

The results of experiment 1 are summarized in Table 2 .

The incidence of polar body extrusion after IVM was lower in the Cd-group compared to the Ctr$\operatorname{group}(P<0.05)$.

Supplementation of Res $1 \mu \mathrm{mol} / \mathrm{L}$ enhanced the maturation rate of oocytes compared to the Cd-group $(P<0.05)$. No difference was recorded among the Cd Res 2-group and other groups.

Cd exposure during IVM reduced the normal fertilization rate $(P<0.05)$ and increased the percentage of oocytes showing more than $2 \mathrm{PN}(P<0.01)$ compared to the Ctr-group. Res, at both tested concentrations, enhanced the occurrence of $2 \mathrm{PN}$ formation $(P<0.05)$ and decreased the polyspermic fertilization compared to the Cd-group $(P<0.01)$.

In Fig.1 representative micrographs of normal (Fig. 1A), polyspermic (Fig. 1B) and asynchronous fertilization (Fig. 1C) after IVF of oocytes matured in presence of $\mathrm{Cd}$ and Res are displayed.

Table 2 Effect of Res supplementation during IVM on meiotic maturation and fertilization of Cdexposed oocytes

\begin{tabular}{|c|c|c|c|c|c|c|}
\hline \multirow[b]{2}{*}{ Groups } & \multirow{2}{*}{$\begin{array}{l}\mathrm{N}^{\circ} \text { of } \\
\text { oocytes }\end{array}$} & \multirow{2}{*}{$\begin{array}{l}\mathbf{N}^{\circ} \text { of } \\
\text { MII oocytes }\end{array}$} & \multicolumn{4}{|c|}{$\mathbf{N}^{\circ}$ of fertilized oocytes $(\%)$} \\
\hline & & & $\begin{array}{l}\text { Normal } \\
\text { (2PNs) }\end{array}$ & $\begin{array}{l}\text { Polyspermy } \\
\text { (> 2PNs) }\end{array}$ & Asynchronous & Unfertilized \\
\hline $\mathrm{Ctr}$ & 120 & $105(87.5 \%)^{\mathrm{a}}$ & $59(56.2 \%)^{\mathrm{a}}$ & $23(21.9 \%)^{\mathrm{b}}$ & $6(4.8 \%)$ & $17(16.2 \%)$ \\
\hline $\mathrm{Cd}$ & 146 & $106(72.6 \%)^{b}$ & $39(36.8 \%)^{b}$ & $38(35.8 \%)^{\mathrm{c}}$ & $7(6.6 \%)$ & $22(20.7 \%)$ \\
\hline Cd-Res 1 & 105 & $88(83.8 \%)^{\mathrm{a}}$ & $47(53.4 \%)^{\mathrm{a}}$ & $16(18.2 \%)^{\mathrm{b}}$ & $7(7.9 \%)$ & $18(20.4 \%)$ \\
\hline Cd-Res 2 & 102 & $80(78.4 \%)^{a b}$ & $41(51.2 \%)^{\mathrm{a}}$ & $13(16.2 \%)^{\mathrm{b}}$ & $6(7.5 \%)$ & $20(25.0 \%)$ \\
\hline
\end{tabular}


The rate of fertilization was calculated from the number of MII oocytes. Values with different superscripts $(\mathrm{a}, \mathrm{b}$, and $\mathrm{c})$ in the same column are significantly different (a vs b: $P<0.05$; b vs. c: $P<$ $0.01)$.

\section{Experiment 2}

\section{The effect of Res on ROS levels in Cd-exposed oocytes}

As shown in Fig. 2, the fluorescence intensity of ROS was higher in the Cd-group than the Ctr-group $(122.4 \pm 1.5, \mathrm{n}=70$ vs. $71.1 \pm 2.3, \mathrm{n}=75 ; P<0.05$; Fig. 2 A and B). Res supplementation reduced ROS levels (74.4 $\pm 1.3, \mathrm{n}=73 ; P<0.05$, Fig. $2 \mathrm{~A}$ and $\mathrm{B})$ as compared to the $\mathrm{Cd}$-group reaching a value comparable to Ctr oocytes.

\section{The effect of Res on mitochondrial distribution and activity in Cd-exposed oocytes}

After IVM, $73.1 \%$ of oocytes of the Ctr-group (n=38/52) showed a homogeneous mt distribution pattern with small granulations spread throughout the cytoplasm (normal distribution, Fig. 3A and C). This pattern was severely altered in oocytes of the Cd-group which exhibited an abnormal mt disposition with medium/large granulations located in the specific cytoplasmic area (Fig. 3C). The proportion of oocytes with normal $\mathrm{mt}$ distribution in the Cd-group was lower $(44.1 \%$; $=26 / 59, P<$ 0.01) than that of the Ctr-group (Fig. 3A). Res treatment restored the rate of normal mt distribution (78.3\%, n=47/60; Fig. 3A and C) with a value higher than in the Cd-group $(P<0.01)$ and similar to the Ctr-group.

Mitochondria activity was higher in the Cd-group than the Ctr-group $(103.1 \pm 1.4$; $n=59$ vs. $67 \pm 0.6$, $\mathrm{n}=52, P<0.01 ;$ Fig.3B). Res supplementation decreased the mt activity $(79.3 \pm 1.1, \mathrm{n}=60 ; P<0.01)$ to a level similar to that of the Ctr oocytes and lower than that of the the Cd-group (Fig. 3B). 


\section{The effect of Res on chromatin and cytoskeleton morphology in Cd-exposed oocytes}

Figure 4 reports the results of LSCM analysis of chromatin, spindle and cortical F-actin of oocytes from Ctr, Cd and Cd-Res groups.

The organization of chromosomes did not differ among groups (Fig. 4A and B).

The meiotic spindle in oocytes from the Ctr group had a classical barrel-shape structure Fig. 4B. The rate of oocytes with normal meiotic spindle structure decreased in the Cd group $(66.7 \%, \mathrm{n}=32 / 48)$ compared to the rate in the $\mathrm{Ctr}$ group $(86.3 \%, \mathrm{n}=44 / 51 ; P<0.05$, Fig. 4A). Res supplementation enhanced the percentage of oocytes with normal spindle structures $(85.7 \%, \mathrm{n}=42 / 49, P<0.05)$ compared to those of the Cd-group reaching a value comparable to the level in the Ctr-group (Fig. 4A).

The oocytes in the Ctr-group displayed a normal cortical F-actin network beneath the oolemma (Fig. 4C). However, the actin filament was abnormally distributed in the Cd-group oocytes which displayed an irregular, discontinuous F-actin staining in the cortical area or a spotted staining within the oocyte cytoplasm (Fig. 4C). The percentage of oocytes with a normal pattern of F-actin organization was lower in the Cd-group $(68.7 \%, \mathrm{n}=33 / 48)$ as compared to the Ctr-group $(88.2 \%, \mathrm{n}=45 / 51 ; P<0.05$, Fig. 4A). Res treatment partially restored the normal F-actin configuration with a rate $(81.6 \%$, n=40/49; Fig. 4A) not statistically different from the Cd-group but similar to Ctr oocytes.

\section{The effect of Res on cortical granules distribution in Cd-exposed oocytes}

Most of Ctr oocytes (94.4\%, n=34/36, Fig. 5A) displayed the CGs localized beneath the oolemma (normal distribution, Fig. 5B). On the contrary, the Cd-group showed an aberrant distribution of CGs which were diffused through the oocyte cytoplasm (Fig. 5B). The percentage of oocytes with a normal pattern of CGs distribution was lower in the Cd-group $(64.7 \%, \mathrm{n}=22 / 34)$ than in the Ctr-group $(P<$ 0.01, Fig. 5A). Res supplementation increased the rate of oocyte with normal distribution of CGs (90.6\%, n=29/32; Fig. 5A and B) as compared to the Cd-group reaching a value similar to the one in the Ctr-group. 


\section{The effect of Res on gene expression levels in Cd-exposed oocytes}

In Fig.6, the expression analysis of specific genes related to oocyte response to oxidative stress is shown. In the Cd-group the expression of SIRT-1, SODI and GPXI genes significantly decreased while GSR gene expression was up-regulated as related to the control $(P<0.05)$.

Res treatment increased the transcript levels of SIRT1 when compared to the Ctr and Cd groups $(P<$ $0.05)$ and up-regulated the expression of SOD1 as related to Cd-group $(P<0.05)$. The expression of GPX1 gene in the Cd-Res group reached a value comparable to the level in the Ctr-group. In the CdRes group the expression of GSR gene was similar as compared to that of the Cd-group and higher $(P<0.05)$ than the Ctr-group. The relative gene expression levels of $C A T$ gene did not differ among groups.

\section{Discussion}

Cadmium is a highly toxic environmental pollutant that can accumulate in female reproductive organs, such as the ovaries, and it can reduce female fertility by impairing oocyte quality. The molecular mechanism by which the adverse effect of $\mathrm{Cd}$ is produced on the female gamete and the toxicity targets have not been fully identified. However, the induction of oxidative stress has been indicated as one of the major factors which may be responsible for Cd-induced deterioration of oocyte quality and function [13]. Studies have shown that, both in in vivo and in vitro animal models, exposure of oocytes to $\mathrm{Cd}$ induced an excessive increase of ROS levels that led to a defective nuclear and cytoplasmic maturation and to an abnormal fertilization $[12,13,22]$. In a previous study, we also proved that the adverse effect of Cd may occur at concentrations lower than previously demonstrated. Indeed, exposure to nanomolar Cd concentrations during IVM impaired in vitro fertilization of sheep oocytes through oxidative damage [8].

In the present study, we investigated the potential beneficial effect of the antioxidant resveratrol against the toxic effects of $\mathrm{Cd}$ exposure on in vitro maturation and fertilization of ovine oocytes. 
Our results confirmed that $\mathrm{Cd}$ caused a defective nuclear and cytoplasmic maturation and impaired normal fertilization in ovine oocytes, in line with previous data in other animal models $[19,21,22]$. More importantly, the main findings of the research proved that Res effectively mitigated Cd-induced alterations of oocyte meiotic and cytoplasmic maturation and restored normal fertilization by reducing ROS accumulation, preventing $\mathrm{mt}$ dysfunction, maintaining the correct cytoskeleton assembly and CGs distribution and modulating the expression of genes involved in oxidative stress response.

In the first part of the study we explored the effect of supplementing IVM medium with two different Res concentrations ( 1 and $2 \mu \mathrm{mol} / \mathrm{L}$ ) on meiotic maturation and fertilization of Cd-exposed oocytes. We found that treatment with Res $1 \mu \mathrm{mol} / \mathrm{L}$ was effective to recover the reduced oocyte meiotic competence induced by $\mathrm{Cd}$ exposure. Moreover, Res, at both tested concentrations, maintained the oocyte ability to be normally fertilized with corresponding decrease of polyspermic fertilization. These results suggested that Res is able to preserve oocytes against deterioration of their quality and function causing a defective maturation and fertilization.

Subsequently, to explore the underling mechanism of Res effect, we evaluated the oxidative and mitochondrial status of in vitro matured oocytes.

It has been well documented that $\mathrm{Cd}$ induced a prolonged generation of ROS by mechanisms involving the depletion of cells' major antioxidants and bonding to sulfhydryl groups of proteins [50, 51]. Another recognized detrimental effect of $\mathrm{Cd}$ is mitochondrial damage [52]. Indeed, Cd-induced excessive ROS can cause disruption of the mitochondrial electron transport with a consequent increase of mitochondrial ROS and cellular oxidative damage [53]. Mitochondria play a vital function in oocyte metabolism and redox homeostasis and provide energy for oocyte maturation, fertilization and embryo development [54]. The distribution pattern of mitochondria and their activity are considered key indicators of oocyte cytoplasmic maturation [55].

Our findings indicated that $\mathrm{Cd}$ exposure caused a significantly increase of ROS production, modifications of the normal mitochondria distribution and over-activity of mitochondria in in vitro 
matured oocytes, in line with our previous results. [8]. Treatment with Res was effective to eliminate ROS overproduction. In addition, this antioxidant prevented defective translocation of mitochondria and regulated their activity recovering a condition similar to controls. These results are consistent with the previous studies which showed the beneficial influence of Res to protect oocytes from oxidative stress by ROS scavenging and to prevent mt dysfunction in standard in vitro embryo production protocols [56], during oocyte in vitro aging [31-33] or after oocyte exposition to toxic substances $[34,35]$.

The correct organization and dynamic of cytoskeleton components are a crucial requisite to drive meiotic progression and polar body extrusion during oocyte maturation [57].

Our data showed that Cd-exposure perturbed the assembly of spindle structure, and impaired the localization of F-actin on the oocyte plasma membrane. Res treatment mitigated $\mathrm{Cd}$ induced disturbance of the meiotic spindle and partially restored the distribution of F-actin in the oocyte cortex. Therefore, we speculate that the protective effect of Res against Cd-induced ROS overproduction and mt dysfunction might be reflected in the maintenance of the correct cytoskeleton assembly with subsequent normal rate of nuclear maturation.

Our study also revealed that Res ameliorated the fertilization capability of Cd exposed oocytes and prevented polyspermic fertilization. The distribution of CGs beneath the oolemma in matured oocytes and exocytosis of their content after fertilization are crucial steps for the blocking of polyspermic fertilization [58]. We demonstrated that the exposure to Cd during IVM disturbed CGs redistribution in the cortex of the oocyte and that Res ensured normal dynamic and localization of CGs, therefore avoiding polyspermic fertilization. According to our findings, Res improved the correct CGs distribution before in vitro fertilization of bovine oocytes [59] and during in vitro aging of porcine oocytes [31].

The protective effect of Res on CGs migration and fertilization might derived from the maintenance of proper mt function and microfilament dynamic which are involved in the process of CGs migration and exocytosis [58]. 
Other important findings of this study concern our transcriptional experiments that were addressed to measure the mRNA levels of SIRT1 and the antioxidant genes SOD1, GPX1, GSR and CAT known to be involved in cellular response to oxidative stress [60].

Specifically, we detected lower expression of SIRT-1, SOD1 and GPX transcripts in Cd-exposed oocytes and the use of Res reversed this effect enhancing the mRNA transcript levels of these genes. Cd induced suppression of SIRT1 signaling has been demonstrated in several cell lines determining oxidative stress, apoptosis, mt and metabolic dysfunctions [61].

It is well documented that Res activates molecular targets involved in endogenous antioxidant system regulation [62]. One of the main pathways of Res action is mediated by SIRT1, a NAD-dependent deacetylase which acts as regulator of the redox state in the oocytes providing protection against oxidative stress [63].

Previous studies showed that Res induced up-regulation of SIRTlgene and antioxidant enzyme genes including SOD1 and GPX1 in bovine oocytes [64] and mouse embryos [65].

In this study, Res treatment enhanced the expression of SIRT1, SOD1 and GPX genes, thus supporting the notion that Res might have counteracted Cd-toxicity by up-regulating SIRT1 and other antioxidant genes such as, SOD1 and GPX1. Interestingly, SIRT-1 gene expression level was significantly higher in Res treated oocytes compared to Ctr oocytes. This evidence suggests that the activation of SIRT1 pathway may play a key role underlying the Res induced response against $\mathrm{Cd}$ toxicity on oocyte maturation.

\section{Conclusions}

In summary, our results demonstrated that Res supplementation during IVM of Cd-exposed ovie oocytes can reverse the $\mathrm{Cd}$ induced oxidative stress by directly scavenging ROS and/or promoting the activity of molecular targets involved in the regulation of the endogenous antioxidant defence. The ameliorative influence of Res was reflected in improvement of mitochondrial function, cytoskeleton morphology, CGs distribution and up-regulating SIRT1, SOD1, GPX1, and ultimately 
in the enhancement of oocyte maturation and fertilization. These findings may provide a useful therapeutic approach for developing protection strategies against $\mathrm{Cd}$-induced toxicity on the female gamete. To thoroughly explore the underlying mechanisms of Res protection, further studies should focus on the assessment of SIRT 1 pathway modulators and antioxidant enzymes activity and on the evaluation of Res effect during IVM of Cd exposed oocytes on cleavage and blastocyst formation.

\section{FIGURE LEGENDS}

Fig. 1 Representative photomicrographs of nuclear configuration of juvenile sheep oocytes $16 \mathrm{~h}$ postin vitro fertilization; A) zygote with two PNs and two PBs (normal fertilization); B) polyspermic fertilized oocyte with three PNs and two PBs and c) asynchronous fertilized oocyte with one PN, an intact sperm head (SH) and two PBs. PN: arrow; PB: asterisk; SH : dot Scale bar $=25 \mu \mathrm{m}$.

Fig. 2 Effect of Res on the intracellular ROS levels in Cd-exposed oocytes. A) Quantification of fluorescence intensity after incubation with the DCHFDA probe. B) Representative LSCM photomicrographs of ROS levels (green) in oocytes from Ctr, Cd and Cd-Res groups. The data are expressed as mean \pm standard error. AU: arbitrary units. Values with different superscripts $(a, b)$ are significantly different $(\mathrm{P}<0.01)$. Scale bar $=25 \mu \mathrm{m}$.

Fig. 3 Effect of Res on the distribution and activity of mitochondria in Cd-exposed oocytes. Graphs showing A) the percentage of oocytes with normal and abnormal mt distribution pattern; B) quantification od fluorescence intensity after incubation with MitoTracker Orange; C) Representative LSCM photomicrographs of $\mathrm{mt}$ distribution (red) in oocytes from $\mathrm{Ctr}, \mathrm{Cd}$ and $\mathrm{Cd}-\mathrm{Res}$ groups. The data are expressed as mean \pm standard error. AU: arbitrary units. Values with different superscripts $(\mathrm{a}, \mathrm{b})$ are significantly different $(\mathrm{P}<0.01)$. Scale bar $=25 \mu \mathrm{m}$.

Fig. 4 Effect of Res on chromatin and cytoskeleton morphology in Cd-exposed oocytes. A) Graph showing the percentage of oocytes with normal and abnormal chromatin, meiotic spindle and F-actin configuration. Representative LSCM images of B) chromosomes (blue) and meiotic spindle (green) 
and C) actin filaments (red) in oocytes of Ctr, Cd and Cd-Res groups. Values with different superscripts $(\mathrm{a}, \mathrm{b})$ are significantly different $(\mathrm{P}<0.05)$. Scale bar $=10 \mu \mathrm{m}$.

Fig. 5 Effect of resveratrol on CGs distribution in Cd-exposed oocytes. A) Graph showing the percentage of oocytes with normal and abnormal CGs distribution and B) representative images of CGs (green) localization in Ctr, Cd and Cd-Res oocytes. Values with different superscripts (a, b) are significantly different $(\mathrm{P}<0.05)$. Scale bar $=25 \mu \mathrm{m}$.

Fig. 6 Effect of Res on relative expression of SIRT 1, SOD1, GPX1, GSR and CAT genes in Ctr, $\mathrm{Cd}$ and Cd-Res oocytes. The data are expressed as mean \pm standard deviation. Values with different superscripts $(\mathrm{a}, \mathrm{b}, \mathrm{c})$ are significantly different $(\mathrm{P}<0.05)$.

\section{Abbreviations}

Cd: Cadmium

Res: Resveratrol

IVM: In vitro maturation

CdCl2: Cadmium chloride

Mt: Mitochondria

ROS: Reactive oxygen species

CGs: Cortical granules

SIRT1: Sirtuin 1

SOD1: Superoxide dismutase 1

GPX1: Glutathione peroxidase 1

GSR: Glutathione-disulphide reductase

CAT: Catalase

IVF: In vitro Fertilization

PBS: Phosphate-buffered saline

COCs: Cumulus-oocyte complexes 
DM: Dissection medium

OSS: Oestrous sheep serum

FSH: Follicle-Stimulating Hormone

LH: Luteinizing Hormone

MII: metaphase II

SOF: Synthetic Oviductal Fluid

PN: Pronucleus

PB: Polar Body

SH: Sperm head

$\mathrm{H}_{2}$ DCF-DA: 2'7'- dichlorodihydrofluorescein diacetate

BSA: Bovine serum albumin

PFA: Paraformaldehyde

DCF: Dichlorofluorescein

LSCM : Laser-scanning confocal microscope

AU: Arbitrary unit

PNA: Lectin peanut agglutinin

RT-PCR: Reverse transcription-polymerase chain reaction

cDNA: Complementary DNA

ANOVA: Parametric Analysis of Variance 


\section{Declarations}

\section{Ethics approval and consent to participate}

All were performed in accordance with DPR 27/1/1992 (Animal Protection regulations of Italy) in conformity with European Community regulation 86/609. No experimental animals have been used in this work.

\section{Consent for publication}

Not applicable.

\section{Availability of data and materials}

All data generated or analysed during this study are included in this published article.

\section{Competing interests}

The authors declare that they have no competing interests.

\section{Funding}

The present work was funded by Fondazione Banco di Sardegna, FDS 2016 (CUP J86C18000780005 and J86C18000810005)

\section{Authors' contributions}

AP, LB designed the study, analyzed data and wrote the paper. AP, FA, A Maltana, conducted IVM and IVF experiments and LSCM analysis. NM, A Mastrorocco, GL performed gene espression experiments. LB, MED and GGL supervised experiments and performed critical data analysis and manuscript revision. All authors read and approved the final manuscript. 


\section{Acknowledgments}

Not applicable.

\section{References}

1. Canipari R, De Santis L, Cecconi S. Female Fertility and Environmental Pollution. Int J Environ Res Public Health. 2020 Nov 26;17(23):8802. doi: 10.3390/ijerph17238802. PMID: 33256215; PMCID: PMC7730072.

2. Wrzecińska M, Kowalczyk A, Cwynar P, Czerniawska-Piątkowska E. Disorders of the Reproductive Health of Cattle as a Response to Exposure to Toxic Metals. Biology (Basel). 2021 Sep 8;10(9):882. doi: 10.3390/biology10090882. PMID: 34571759; PMCID: PMC8467698.

3. Swaran J.S. Flora, Shruti Agrawal, Chapter 31 - Arsenic, Cadmium, and Lead. Reproductive and Developmental Toxicology (Second Edition), Academic Press, 2017, 537-566. https://doi.org/10.1016/B978-0-12-804239-7.00031-7.

4. Park HJ, Kim SU, Jung KY, Lee S, Choi YD, Owens VN, Kumar S, Yun SW, Hong CO. Cadmium phytoavailability from 1976 through 2016: Changes in soil amended with phosphate fertilizer and compost. Sci Total Environ. 2021 Mar 25;762:143132. doi: 10.1016/j.scitotenv.2020.143132. Epub 2020 Oct 16. PMID: 33121778.

5. Richter P, Faroon O, Pappas RS. Cadmium and Cadmium/Zinc Ratios and Tobacco-Related Morbidities. Int J Environ Res Public Health. 2017 Sep 29;14(10):1154. doi: 10.3390/ijerph14101154. PMID: 28961214; PMCID: PMC5664655.

6. Genchi G, Sinicropi MS, Lauria G, Carocci A, Catalano A. The Effects of Cadmium Toxicity. Int J Environ Res Public Health. 2020 May 26;17(11):3782. doi: 10.3390/ijerph17113782. PMID: 32466586; PMCID: PMC7312803. 
7. Zenzes MT, Krishnan S, Krishnan B, Zhang H, Casper RF. Cadmium accumulation in follicular fluid of women in in vitro fertilization-embryo transfer is higher in smokers. Fertil Steril. 1995 Sep;64(3):599-603. doi: 10.1016/s0015-0282(16)57799-1. PMID: 7641916.

8. Martino NA, Marzano G, Mangiacotti M, Miedico O, Sardanelli AM, Gnoni A, Lacalandra GM, Chiaravalle AE, Ciani E, Bogliolo L, Minervini F, Pizzi F, Dell'Aquila ME. Exposure to cadmium during in vitro maturation at environmental nanomolar levels impairs oocyte fertilization through oxidative damage: A large animal model study. Reprod Toxicol. 2017 Apr;69:132-145. doi: 10.1016/j.reprotox.2017.02.005. Epub 2017 Feb 7. PMID: 28188904.

9. Wdowiak A, Wdowiak E, Bojar I. Evaluation of trace metals in follicular fluid in ICSI-treated patients. Ann Agric Environ Med. 2017 Jun 23;25(2):213-218. doi: 10.26444/aaem/75422. Epub 2017 Jun 23. PMID: 29936824.

10. Wu S, Wang M, Deng Y, Qiu J, Zhang X, Tan J. Associations of toxic and essential trace elements in serum, follicular fluid, and seminal plasma with In vitro fertilization outcomes. Ecotoxicol Environ Saf. 2020 Nov;204:110965. doi: 10.1016/j.ecoenv.2020.110965. Epub 2020 Aug 13. PMID: 32798747.

11. Zhu JQ, Liu Y, Zhang JH, Liu YF, Cao JQ, Huang ZT, Yuan Y, Bian JC, Liu ZP. Cadmium Exposure of Female Mice Impairs the Meiotic Maturation of Oocytes and Subsequent Embryonic Development. Toxicol Sci. 2018 Jul 1;164(1):289-299. doi: 10.1093/toxsci/kfy089. PMID: 29684212.

12. Cheng Y, Zhang J, Wu T, Jiang X, Jia H, Qing S, An Q, Zhang Y, Su J. Reproductive toxicity of acute $\mathrm{Cd}$ exposure in mouse: Resulting in oocyte defects and decreased female fertility. Toxicol Appl Pharmacol. 2019 Sep 15;379:114684. doi: 10.1016/j.taap.2019.114684. Epub 2019 Jul 17. PMID: 31325558.

13. Dong F, Li J, Lei WL, Wang F, Wang Y, Ouyang YC, Hou Y, Wang ZB, Schatten H, Sun QY. Chronic cadmium exposure causes oocyte meiotic arrest by disrupting spindle assembly 
checkpoint and maturation promoting factor. Reprod Toxicol. 2020 Sep;96:141-149. doi: 10.1016/j.reprotox.2020.06.009. Epub 2020 Jun 20. PMID: 32574675.

14. Ruslee SS, Zaid SSM, Bakrin IH, Goh YM, Mustapha NM. Protective effect of Tualang honey against cadmium-induced morphological abnormalities and oxidative stress in the ovary of rats. BMC Complement Med Ther. 2020 May 29;20(1):160. doi: 10.1186/s12906-020-02960-1. PMID: 32471398; PMCID: PMC7260854.

15. Tian J, Hu J, Liu D, Yin J, Chen M, Zhou L, Yin H. Cadmium chloride-induced transgenerational neurotoxicity in zebrafish development. Environ Toxicol Pharmacol. 2021 Jan;81:103545. doi: 10.1016/j.etap.2020.103545. Epub 2020 Nov 7. PMID: 33171223.

16. Thompson J, Bannigan J. Cadmium: toxic effects on the reproductive system and the embryo. Reprod Toxicol. 2008 Apr;25(3):304-15. doi: 10.1016/j.reprotox.2008.02.001. Epub 2008 Feb 19. PMID: 18367374.

17. Leoni G, Bogliolo L, Deiana G, Berlinguer F, Rosati I, Pintus PP, Ledda S, Naitana S. Influence of cadmium exposure on in vitro ovine gamete dysfunction. Reprod Toxicol. 2002 JulAug;16(4):371-77. doi: 10.1016/s0890-6238(02)00040-0. PMID: 12220597.

18. Lazzari G, Tessaro I, Crotti G, Galli C, Hoffmann S, Bremer S, Pellizzer C. Development of an in vitro test battery for assessing chemical effects on bovine germ cells under the ReProTect umbrella. Toxicol Appl Pharmacol. 2008 Dec 15;233(3):360-70. doi: 10.1016/j.taap.2008.08.019. Epub 2008 Sep 16. PMID: 18835402.

19. Nandi S, Gupta PS, Selvaraju S, Roy SC, Ravindra JP. Effects of exposure to heavy metals on viability, maturation, fertilization, and embryonic development of buffalo (Bubalus bubalis) oocytes in vitro. Arch Environ Contam Toxicol. 2010 Jan;58(1):194-204. doi: 10.1007/s00244-0099342-7. Epub 2009 May 28. PMID: 19475365.

20. Tessaro I, Modina SC, Crotti G, Franciosi F, Colleoni S, Lodde V, Galli C, Lazzari G, Luciano AM. Transferability and inter-laboratory variability assessment of the in vitro bovine oocyte 
fertilization test. Reprod Toxicol. 2015 Jan;51:106-13. doi: 10.1016/j.reprotox.2015.01.001. Epub 2015 Jan 25. PMID: 25625651.

21. Akar Y, Ahmad N, Khalıd M. The effect of cadmium on the bovine in vitro oocyte maturation and early embryo development. Int J Vet Sci Med. 2018 Mar 22;6(Suppl):S73-S77. doi: 10.1016/j.ijvsm.2018.03.001. PMID: 30761325; PMCID: PMC6161866.

22. Zhou C, Zhang X, Chen Y, Liu X, Sun Y, Xiong B. Glutathione alleviates the cadmium exposure-caused porcine oocyte meiotic defects via eliminating the excessive ROS. Environ Pollut. 2019 Dec;255(Pt 1):113194. doi: 10.1016/j.envpol.2019.113194. Epub 2019 Sep 7. PMID: 31520902.

23. Massányi M, Satarug S, Madeddu R, Stawarz R, Massányi P. Evidence for Ovarian and Testicular Toxicities of Cadmium and Detoxification by Natural Substances. Stresses. 2022; 2(1):116. https://doi.org/10.3390/stresses2010001

24. Kechiche S, Venditti M, Knani L, Jabłońska K, Dzięgiel P, Messaoudi I, Reiter RJ, Minucci S. First evidence of the protective role of melatonin in counteracting cadmium toxicity in the rat ovary via the mTOR pathway. Environ Pollut. 2021 Feb 1;270:116056. doi: 10.1016/j.envpol.2020.116056. Epub 2020 Nov 9. PMID: 33199064.

25. Repossi G, Das UN, Eynard AR. Molecular Basis of the Beneficial Actions of Resveratrol. Arch Med Res. 2020 Feb;51(2):105-114. doi: 10.1016/j.arcmed.2020.01.010. Epub 2020 Feb 26. PMID: 32111491.

26. Sovernigo TC, Adona PR, Monzani PS, Guemra S, Barros F, Lopes FG, Leal C. Effects of supplementation of medium with different antioxidants during in vitro maturation of bovine oocytes on subsequent embryo production. Reprod Domest Anim. 2017 Aug;52(4):561-569. doi: 10.1111/rda.12946. Epub 2017 Mar 10. PMID: 28295710.

27. Liu MJ, Sun AG, Zhao SG, Liu H, Ma SY, Li M, Huai YX, Zhao H, Liu HB. Resveratrol improves in vitro maturation of oocytes in aged mice and humans. Fertil Steril. 2018 May;109(5):900-907. doi: 10.1016/j.fertnstert.2018.01.020. PMID: 29778389. 
28. Piras AR, Ariu F, Falchi L, Zedda MT, Pau S, Schianchi E, Paramio M, Bogliolo L. Resveratrol treatment during maturation enhances developmental competence of oocytes after prolonged ovary storage at $4{ }^{\circ} \mathrm{C}$ in the domestic cat model. Theriogenology. 2020 Mar 1;144:152157. doi: 10.1016/j.theriogenology.2020.01.009. Epub 2020 Jan 8. PMID: 31951984.

29. Zabihi A, Shabankareh HK, Hajarian H, Foroutanifar S. Resveratrol addition to in vitro maturation and in vitro culture media enhances developmental competence of sheep embryos. Domest Anim Endocrinol. 2019 Jul;68:25-31. doi: 10.1016/j.domaniend.2018.12.010. Epub 2019 Jan 5. PMID: 30784945.

30. Piras AR, Menéndez-Blanco I, Soto-Heras S, Catalá MG, Izquierdo D, Bogliolo L, Paramio MT. Resveratrol supplementation during in vitro maturation improves embryo development of prepubertal goat oocytes selected by brilliant cresyl blue staining. J Reprod Dev. 2019 Apr 12;65(2):113-120. doi: 10.1262/jrd.2018-077. Epub 2018 Dec 29. PMID: 30606957; PMCID: PMC6473110.

31. Ma R, Zhang Y, Zhang L, Han J, Rui R. Sirt1 protects pig oocyte against in vitro aging. Anim Sci J. 2015 Sep;86(9):826-32. doi: 10.1111/asj.12360. Epub 2015 Jan 20. PMID: 25601632.

32. Zhou J, Xue Z, He HN, Liu X, Yin SY, Wu DY, Zhang X, Schatten H, Miao YL. Resveratrol delays postovulatory aging of mouse oocytes through activating mitophagy. Aging (Albany NY). 2019 Dec 13;11(23):11504-11519. doi: 10.18632/aging.102551. Epub 2019 Dec 13. PMID: 31834867; PMCID: PMC6932885.

33. Abbasi B, Dong Y, Rui R. Resveratrol Hinders Postovulatory Aging by Modulating Oxidative Stress in Porcine Oocytes. Molecules. 2021 Oct 20;26(21):6346. doi: 10.3390/molecules26216346. PMID: 34770755; PMCID: PMC8588440.

34. Liu Y, He XQ, Huang X, Ding L, Xu L, Shen YT, Zhang F, Zhu MB, Xu BH, Qi ZQ, Wang HL. Resveratrol protects mouse oocytes from methylglyoxal-induced oxidative damage. PLoS One. 2013 Oct 23;8(10):e77960. doi: 10.1371/journal.pone.0077960. PMID: 24194906; PMCID: PMC3806792. 
35. Han J, Wang H, Zhang T, Chen Z, Zhao T, Lin L, Xia G, Wang C. Resveratrol attenuates doxorubicin-induced meiotic failure through inhibiting oxidative stress and apoptosis in mouse oocytes. Aging (Albany NY). 2020 Apr 30;12(9):7717-7728. doi: 10.18632/aging.103061. Epub 2020 Apr 30. PMID: 32352929; PMCID: PMC7244048.

36. Li Y, Wang J, Zhang Z, Yi J, He C, Wang F, Tian X, Yang M, Song Y, He P, Liu G. Resveratrol compares with melatonin in improving in vitro porcine oocyte maturation under heat stress. J Anim Sci Biotechnol. 2016 Jun 3;7:33. doi: 10.1186/s40104-016-0093-9. PMID: 27274843; PMCID: PMC4891897.

37. Ito J, Shirasuna K, Kuwayama T, Iwata H. Resveratrol treatment increases mitochondrial biogenesis and improves viability of porcine germinal-vesicle stage vitrified-warmed oocytes. Cryobiology. 2020 Apr;93:37-43. doi: 10.1016/j.cryobiol.2020.02.014. Epub 2020 Mar 11. PMID: 32171796.

38. Chinen S, Yamanaka T, Hirabayashi M, Hochi S. Rescue of vitrified-warmed bovine mature oocytes by short-term recovery culture with resveratrol. Cryobiology. 2020 Dec;97:185-190. doi: 10.1016/j.cryobiol.2020.03.004. Epub 2020 Mar 7. PMID: 32156621.

39. Bogliolo L, Ariu F, Leoni G, Uccheddu S, Bebbere D. High hydrostatic pressure treatment improves the quality of in vitro-produced ovine blastocysts. Reprod Fertil Dev. 2011;23(6):809-17. doi: 10.1071/RD11023. PMID: 21791182.

40. Tervit HR, Whittingham DG, Rowson LE. Successful culture in vitro of sheep and cattle ova. J Reprod Fertil. 1972 Sep;30(3):493-7. doi: 10.1530/jrf.0.0300493. PMID: 4672493.

41. Menéndez-Blanco I, Soto-Heras S, Catalá MG, Piras AR, Izquierdo D, Paramio MT. Effect of vitrification of in vitro matured prepubertal goat oocytes on embryo development after parthenogenic activation and intracytoplasmic sperm injection. Cryobiology. 2020 Apr;93:56-61. doi: 10.1016/j.cryobiol.2020.02.011. Epub 2020 Feb 25. PMID: 32109458.

42. Leoni GG, Palmerini MG, Satta V, Succu S, Pasciu V, Zinellu A, Carru C, Macchiarelli G, Nottola SA, Naitana S, Berlinguer F. Differences in the Kinetic of the First Meiotic Division and in 
Active Mitochondrial Distribution between Prepubertal and Adult Oocytes Mirror Differences in their Developmental Competence in a Sheep Model. PLoS One. 2015 Apr 20;10(4):e0124911. doi: 10.1371/journal.pone.0124911. PMID: 25893245; PMCID: PMC4403920.

43. Ariu F, Bogliolo L, Pinna A, Malfatti L, Innocenzi P, Falchi L, Bebbere D, Ledda S. Cerium oxide nanoparticles $\left(\mathrm{CeO}_{2} \mathrm{NPs}\right)$ improve the developmental competence of in vitro-matured prepubertal ovine oocytes. Reprod Fertil Dev. 2017 Apr;29(5):1046-1056. doi: 10.1071/RD15521. PMID: 28442051.

44. Succu S, Leoni GG, Berlinguer F, Madeddu M, Bebbere D, Mossa F, Bogliolo L, Ledda S, Naitana S. Effect of vitrification solutions and cooling upon in vitro matured prepubertal ovine oocytes. Theriogenology. 2007 Jul 1;68(1):107-14. doi: 10.1016/j.theriogenology.2007.04.035. Epub 2007 May 29. PMID: 17537497.

45. Bogliolo L, Murrone O, Piccinini M, Ariu F, Ledda S, Tilocca S, Albertini DF. Evaluation of the impact of vitrification on the actin cytoskeleton of in vitro matured ovine oocytes by means of Raman microspectroscopy. J Assist Reprod Genet. 2015 Feb;32(2):185-93. doi: 10.1007/s10815014-0389-7. Epub 2014 Nov 16. PMID: 25399064; PMCID: PMC4354177.

46. Hosseini SM, Asgari V, Ostadhosseini S, Hajian M, Piryaei A, Najarasl M, Nasr-Esfahani MH. Potential applications of sheep oocytes as affected by vitrification and in vitro aging. Theriogenology. 2012 Jun;77(9):1741-53. doi: 10.1016/j.theriogenology.2011.12.005. Epub 2012 Mar 22. PMID: 22444551.

47. Palmerini MG, Nottola SA, Leoni GG, Succu S, Borshi X, Berlinguer F, Naitana S, Bekmukhambetov Y, Macchiarelli G. In vitro maturation is slowed in prepubertal lamb oocytes: ultrastructural evidences. Reprod Biol Endocrinol. 2014 Nov 24;12:115. doi: 10.1186/1477-7827-12115. PMID: 25421574; PMCID: PMC4247763.

48. Ren J, Hao Y, Liu Z, Li S, Wang C, Wang B, Liu Y, Liu G, Dai Y. Effect of exogenous glutathione supplementation on the in vitro developmental competence of ovine oocytes. 
Theriogenology. 2021 Oct 1;173:144-155. doi: 10.1016/j.theriogenology.2021.07.025. Epub 2021 Aug 8. PMID: 34390905.

49. Livak KJ, Schmittgen TD. Analysis of relative gene expression data using real-time quantitative PCR and the 2(-Delta Delta C(T)) Method. Methods. 2001 Dec;25(4):402-8. doi: 10.1006/meth.2001.1262. PMID: 11846609.

50. Stohs et al. S.J.; Bagchi, D. Oxidative mechanisms in the toxicity of metal ions. Free Radic. Biol. Med. 1995, 18,) Valko, M.; Morris, H.; Cronin, M.T.D. Metals, toxicity and oxidative stress. Curr. Med. Chem. 2005, 12, 1161-1208.

51. Valko M, Morris H, Cronin MT. Metals, toxicity and oxidative stress. Curr Med Chem. 2005;12(10):1161-208. doi: 10.2174/0929867053764635. PMID: 15892631.

52. Li M, Xia T, Jiang CS, Li LJ, Fu JL, Zhou ZC. Cadmium directly induced the opening of membrane permeability pore of mitochondria which possibly involved in cadmium-triggered apoptosis. Toxicology. 2003 Dec 15;194(1-2):19-33. doi: 10.1016/s0300-483x(03)00327-5. PMID: 14636693.

53. Cannino G, Ferruggia E, Luparello C, Rinaldi AM. Cadmium and mitochondria. Mitochondrion. 2009 Nov;9(6):377-84. doi: 10.1016/j.mito.2009.08.009. Epub 2009 Aug 23. PMID: 19706341

54. Ge H, Tollner TL, Hu Z, Dai M, Li X, Guan H, Shan D, Zhang X, Lv J, Huang C, Dong Q. The importance of mitochondrial metabolic activity and mitochondrial DNA replication during oocyte maturation in vitro on oocyte quality and subsequent embryo developmental competence. Mol Reprod Dev. 2012 Jun;79(6):392-401. doi: 10.1002/mrd.22042. Epub 2012 Apr 16. PMID: 2246

55. Kirillova A, Smitz JEJ, Sukhikh GT, Mazunin I. The Role of Mitochondria in Oocyte Maturation. Cells. 2021 Sep 19;10(9):2484. doi: 10.3390/cells10092484. PMID: 34572133; PMCID: PMC8469615. 
56. Galeati G and Spinaci M. Resveratrol from Red Grapes: An Useful Agent for Oocyte Maturation and Subsequent Embryonic Development. Austin J In Vitro Fertili. 2015;2(1): 1014. ISSN:2471-0628

57. Liu XJ. Polar body emission. Cytoskeleton (Hoboken). 2012 Oct;69(10):670-85. doi: 10.1002/cm.21041. Epub 2012 Jun 21. PMID: 22730245.

58. Liu M. The biology and dynamics of mammalian cortical granules. Reprod Biol Endocrinol. 2011 Nov 17;9:149. doi: 10.1186/1477-7827-9-149. PMID: 22088197; PMCID: PMC3228701.

59. Takeo S, Sato D, Kimura K, Monji Y, Kuwayama T, Kawahara-Miki R, Iwata H. Resveratrol improves the mitochondrial function and fertilization outcome of bovine oocytes. J Reprod Dev. 2014 Apr 24;60(2):92-9. doi: 10.1262/jrd.2013-102. Epub 2013 Dec 27. PMID: 24390595; PMCID: PMC3999399.

60. Tatone C, Di Emidio G, Barbonetti A, Carta G, Luciano AM, Falone S, Amicarelli F. Sirtuins in gamete biology and reproductive physiology: emerging roles and therapeutic potential in female and male infertility. Hum Reprod Update. 2018 May 1;24(3):267-289. doi: 10.1093/humupd/dmy003. PMID: 29447380.

61. Tinkov AA, Nguyen TT, Santamaria A, Bowman AB, Buha Djordjevic A, Paoliello MMB, Skalny AV, Aschner M. Sirtuins as molecular targets, mediators, and protective agents in metalinduced toxicity. Arch Toxicol. 2021 Jul;95(7):2263-2278. doi: 10.1007/s00204-021-03048-6. Epub 2021 May 24. PMID: 34028595.

62. Xia N, Daiber A, Förstermann U, Li H. Antioxidant effects of resveratrol in the cardiovascular system. Br J Pharmacol. 2017 Jun;174(12):1633-1646. doi: 10.1111/bph.13492. Epub 2016 May 6. PMID: 27058985; PMCID: PMC5446570.

63. Tatone, C., Di Emidio, G., Vitti, M., Di Carlo, M., Santini, S., Jr, D'Alessandro, A. M., Falone, S., \& Amicarelli, F. (2015). Sirtuin Functions in Female Fertility: Possible Role in Oxidative Stress and Aging. Oxidative medicine and cellular longevity, 2015, 659687. https://doi.org/10.1155/2015/659687 
64. Wang F, Tian X, Zhang L, He C, Ji P, Li Y, Tan D, Liu G. Beneficial effect of resveratrol on bovine oocyte maturation and subsequent embryonic development after in vitro fertilization. Fertil Steril. 2014 Feb;101(2):577-86. doi: 10.1016/j.fertnstert.2013.10.041. Epub 2013 Dec 5. PMID: 24314921.

65. Lin C, Yon JM, Jung AY, Lee JG, Jung KY, Kang JK, Lee BJ, Yun YW, Nam SY. Resveratrol prevents nicotine-induced teratogenesis in cultured mouse embryos. Reprod Toxicol. 2012 Nov;34(3):340-6. doi: 10.1016/j.reprotox.2012.05.097. Epub 2012 Jun 9. PMID: 22687363. 


\section{Figures}

\section{Figure 1}

Representative photomicrographs of nuclear configuration of juvenile sheep oocytes $16 \mathrm{~h}$ post $\mathbb{\text { in }}$ vitro fertilization; A) zygote with two PNs and two PBs (normal fertilization); B) polyspermic fertilized oocyte with three PNs and two PBs and c) asynchronous fertilized oocyte with one PN, an intact sperm head (SH) and two PBs. PN: arrow; PB: asterisk; $\mathrm{SH}$ : dot Scale bar $=25 \mu \mathrm{m}$.

\section{Figure 2}

Effect of Res on the intracellular ROS levels in Cd-exposed oocytes. A) Quantification of fluorescence intensity after incubation with the DCHFDA probe. B) Representative LSCM photomicrographs of ROS levels (green) in oocytes from $\mathrm{Ctr}, \mathrm{Cd}$ and $\mathrm{Cd}$-Res groups. The data are expressed as mean \pm standard error. AU: arbitrary units. Values with different superscripts $(a, b)$ are significantly different $(P<0.01)$. Scale bar $=25 \mu \mathrm{m}$.

\section{Figure 3}

Effect of Res on the distribution and activity of mitochondria in Cd-exposed oocytes. Graphs showing A) the percentage of oocytes with normal and abnormal mt distribution pattern; B) quantification od fluorescence intensity after incubation with MitoTracker Orange; C) Representative LSCM photomicrographs of $\mathrm{mt}$ distribution (red) in oocytes from Ctr, $\mathrm{Cd}$ and $\mathrm{Cd}-\mathrm{Res}$ groups. The data are expressed as mean \pm standard error. AU: arbitrary units. Values with different superscripts $(a, b)$ are significantly different $(P<0.01)$. Scale bar $=25 \mu \mathrm{m}$.

\section{Figure 4}

Effect of Res on chromatin and cytoskeleton morphology in Cd-exposed oocytes. A) Graph showing the percentage of oocytes with normal and abnormal chromatin, meiotic spindle and F-actin configuration. Representative LSCM images of B) chromosomes (blue) and meiotic spindle (green) and C) actin filaments (red) in oocytes of $\mathrm{Ctr}, \mathrm{Cd}$ and $\mathrm{Cd}$-Res groups. Values with different superscripts $(\mathrm{a}, \mathrm{b})$ are significantly different $(P<0.05)$. Scale bar $=10 \mu \mathrm{m}$. 


\section{Figure 5}

Effect of resveratrol on CGs distribution in Cd-exposed oocytes. A) Graph showing the percentage of oocytes with normal and abnormal CGs distribution and B) representative images of CGs (green) localization in $\mathrm{Ctr}$, $\mathrm{Cd}$ and $\mathrm{Cd}$-Res oocytes. Values with different superscripts $(\mathrm{a}, \mathrm{b})$ are significantly different $(P<0.05)$. Scale bar $=25 \mu \mathrm{m}$.

\section{Figure 6}

Effect of Res on relative expression of SIRT 1, SOD1, GPX1, GSR and CAT genes in Ctr, Cd and Cd-Res oocytes. The data are expressed as mean \pm standard deviation. Values with different superscripts $(a, b, c)$ are significantly different $(P<0.05)$. 